\title{
Reversible posterior leukoencephalopathy syndrome in a patient with systemic lupus erythematosus; a diagnostic dilemma
}

\author{
Udugama JMM, Lekamwasam JDVC, Vijayabandara MSK, Chathurani MMI, Abeyratne WKDT \\ Teaching Hospital, Karapitiya, Galle, Sri Lanka.
}

Correspondence: Dr. JMM Udugama

e-mail:malikaudugama06@gmail.com

iD https://orcid.org/0000-0002-8550-013X

\begin{abstract}
Introduction
Reversible posterior leucoencephalopathy syndrome (RPLS) is a clinical radiographic syndrome of a multitude of etiologies. It is characterized by headache, visual disturbances, seizures, altered mental status, and radiological findings secondary to brain edema predominantly in areas supplied by posterior circulation. It is often, but by no means always, associated with an acute raise of blood pressure in patients with hypertension. RPLS should be promptly recognized as it is a reversible and treatable condition. Here we report a case of RPLS in a normotensive patient with systemic lupus erythematosus (SLE)/secondary anti phospholipid syndrome (APLS).
\end{abstract}

\section{Case report}

A 43 year- old female with SLE and secondary APLS complicated with deep vein thrombosis presented to the ward with epigastric pain and dyspeptic symptoms for 3 days. She had been on prednisolone and warfarin without a proper follow up and had been off prednisolone for 2 weeks prior to admission. Her symptoms subsided and she was awaiting discharge, when she complained of generalized headache and intermittent blurring of vision. A few hours later she developed generalized tonic clonic convulsions with frothing and urinary incontinence. Following convulsions she developed confusion and altered behavior. On physical examination, she was drowsy and confused, with no neck stiffness. There were no features of active SLE such as synovitis, dermatological manifestations or serositis. Blood pressure was 120/70 mmHg.

According to the above findings, the differential diagnoses of cerebral infection, neuropsychiatric lupus, cerebral venous thrombosis and intracranial haemorrhage were considered. Subsequent investigations revealed a white cell count of $11.0 \times 10^{3} / \mathrm{mL}$ (neutrophils $70 \%$ ), platelets 191,000, CRP and ESR were $42 \mathrm{mg} / \mathrm{dL}$ and $56 \mathrm{~mm}$, respectively. CSF full report revealed 3 polymorphs, 10 lymphocytes, increased protein $(60 \mathrm{mg} / \mathrm{dL})$, and normal sugar levels. CSF culture revealed no growth. Serum calcium levels and electrolytes were normal and her non-contrast CT brain did not reveal any abnormality. EEG showed generalized slow waves with delta activity throughout the recording with no epileptiform activity.

She was started on IV ceftriaxone 2 g, IV vancomycin $1 \mathrm{~g}$ and IV acyclovir with concurrent administration of IV methylprednisolone $1 \mathrm{~g} /$ day pulses. The patient made a remarkable recovery within 2 days of symptom onset. Since the CSF report was inconclusive MRI / MRV was carried out to arrive at a definite diagnosis (Figure $1 \& 2$ ). The MRI FLAIR revealed subcortical T2 hyperintensity without enhancement on both occipital lobes (Figure 1). With these findings antibiotics were omitted.

\section{Discussion}

RPLS has been reported in literature as a reversible yet underdiagnosed manifestation of neuropsychiatric lupus (6). The pathophysiology of SLE related RPLS is incompletely understood, but cerebral auto regulatory failure and endothelial dysfunction causing disruption of the blood brain barrier leading to fluid transudation into the brain have been postulated (6). 


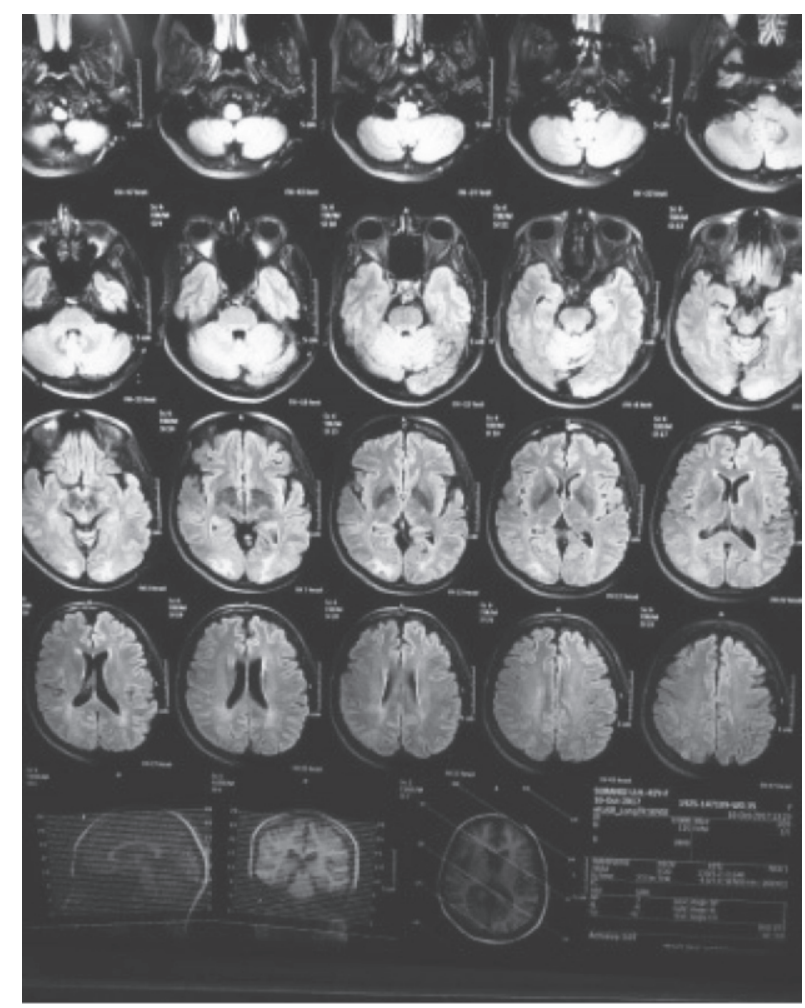

Figure 1: MRI flare image showing high signal intensity in both occipital lobes

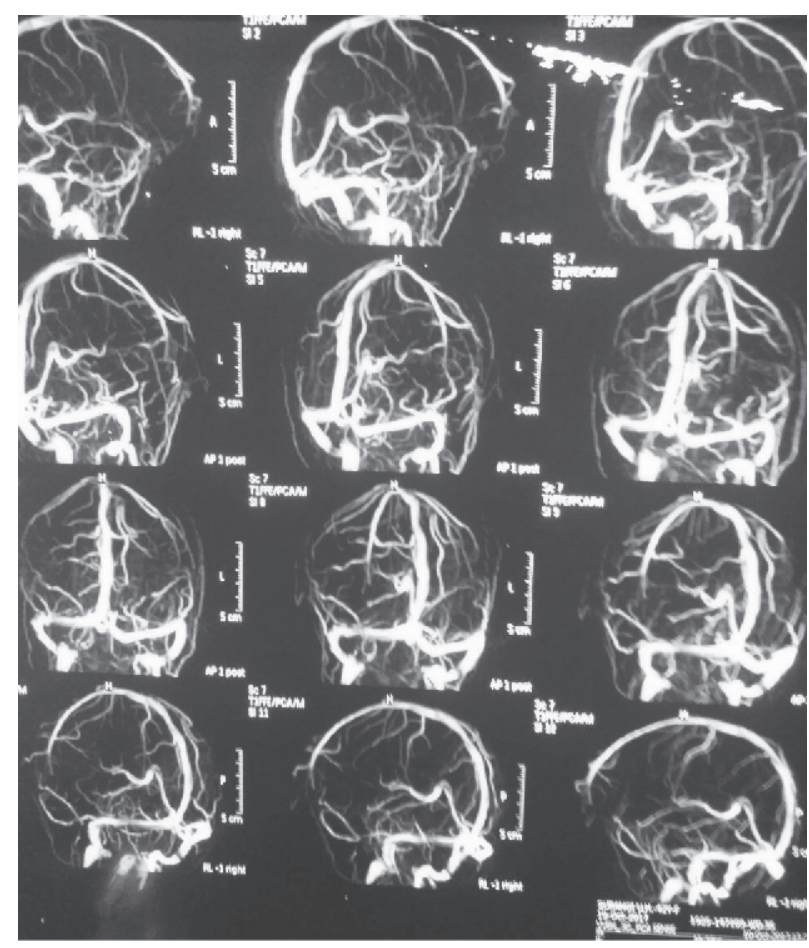

Figure 2: Normal MRV brain, done to exclude cerebral venous thrombosis
In many case series conducted on SLE related RPLS acute hypertension, high SLE disease activity, renal involvement and the use of immunosuppressive agents, particularly cyclosporine have been identified as risk factors (3). RPLS occurring in a normotensive SLE patient, in the absence of accompanying lupus nephritis, features of high disease activity and immunosuppressants has rarely been reported in literature.

In a patient with SLE, the diagnostic dilemma lies in the differentiation of RPLS from cerebral infections due to immunosuppressants and from thrombotic events due to APLS. In cases where cerebrospinal fluid sampling was carried out, elevated protein was the most common finding. Though CSF pleocytosis was uncommon, it had also been reported and its presence should not exclude the possibility of RPLS. (4). Cerebral imaging is the mainstay of diagnosis and in most cases CT can be normal though it is useful in ruling out arterial ischaemia or thrombosis.

In RPLS, MRI is the investigation with the most diagnostic value and typical MRI findings include bilateral white-matter abnormalities in vascular watershed areas in the posterior regions of both cerebral hemispheres, affecting mostly the occipital and parietal lobes. Atypical features including hemorrhage, asymmetrical changes, isolated involvement of the frontal lobes, and cortical lesions are also common (5).

The management of RPLS in SLE is dependent on aetiology. Since hypertension is a feature in the majority of patients, they often improve dramatically with rapid blood pressure lowering. Seizures are usually treated with phenytoin and in cases associated with cytotoxic drugs prompt removal or dosage reduction of the offending agent is recommended. In the rare instance, such as this case, where SLE per se acted as the trigger for RPLS, vigorous treatment with corticosteroids has been suggested. Nevertheless, it is still controversial whether immunosuppression should be initiated in RPLS in SLE and proper treatment strategies are yet to be instigated.

\section{Consent}

Written informed consent was obtained from the patient for publication of this case report and accompanying images 


\section{Conflict of Interests}

The authors declare that there is no conflict of interests regarding the publication of this paper.

\section{References}

1. Fugate JE, Claassen DO, Cloft HJ, et al. Posterior reversible encephalopathy syndrome: associated clinical and radiologic findings. Mayo Clin Proc, 2010; 85: 427-32.

2. Juliana Gomez, Erik J. Jhonston, Francisco Zevallos. Posterior Reversible Encephalopathy Syndrome in a Patient with Systemic Lupus Erythematosus / Systemic Sclerosis Overlap Syndrome. Case Reports in Rheumatology, Volume 2014.

3. Kur JK, Esdaile JM. Posterior reversible encephalopathy syndrome - an under recognized manifestation of systemic lupus erythematosus. J Rheumatol, 2006; 33(11): 2178-83.
4. Colin Ellis, Ramani Balu.Cerebrospinal Fluid Characteristics in Patients with Posterior Reversible Encephalopathy Syndrome (PRES). Neurology, April 05, 2016; 86 (16 Supplement).

5. Esther V. Hobson, Ian Craven, et al. Reversible Encephalopathy Syndrome: A Truly Treatable Neurologic Illness. Perit Dial Int, 2012 Nov-Dec; 32(6): 590-4.

6. Bin Liu, Yuan Yao, Feng Chung Zang. Posterior reversible encephalopathy syndrome could be an underestimated variant of "reversible neurological deficits" in Systemic Lupus Erythematosus. BMC Neurology, 2012;12: 152. 